\title{
OPEN Glial cells modulate retinal cell survival in rotenone-induced neural degeneration
}

\author{
Hiroshi Tawarayama $a^{1,2,6}$, Maki Inove-Yanagimachi ${ }^{1,6}$, Noriko Himori ${ }^{1} \&$ \\ Toru Nakazawa ${ }^{1,2,3,4,5 \boxplus}$
}

Administration of the mitochondrial complex I inhibitor rotenone provides an excellent model to study the pathomechanism of oxidative stress-related neural degeneration diseases. In this study, we examined the glial roles in retinal cell survival and degeneration under the rotenone-induced oxidative stress condition. Mouse-derived Müller, microglial (BV-2), and dissociated retinal cells were used for in vitro experiments. Gene expression levels and cell viability were determined using quantitative reverse transcription-polymerase chain reaction and the alamarBlue assay, respectively. Conditioned media were prepared by stimulating glial cells with rotenone. Retinal ganglion cells (RGCs) and inner nuclear layer (INL) were visualized on rat retinal sections by immunohistochemistry and eosin/hematoxylin, respectively. Rotenone dose-dependently induced glial cell death. Treatment with rotenone or rotenone-stimulated glial cell-conditioned media altered gene expression of growth factors and inflammatory cytokines in glial cells. The viability of dissociated retinal cells significantly increased upon culturing in media conditioned with rotenone-stimulated or Müller cell-conditioned media-stimulated BV-2 cells. Furthermore, intravitreal neurotrophin- 5 administration prevented the rotenone-induced reduction of RGC number and INL thickness in rats. Thus, glial cells exerted both positive and negative effects on retinal cell survival in rotenone-induced neural degeneration via altered expression of growth factors, especially upregulation of microglia-derived Ntf5, and proinflammatory cytokines.

Rotenone, a natural compound found in the roots and stems of certain plants, acts as a strong inhibitor of nicotinamide adenine dinucleotide (NAD)-ubiquinone oxidoreductase of the mitochondrial respiratory chain ${ }^{1,2}$. Rotenone inhibits electron transfer from iron-sulfur centers in mitochondrial complex I to oxygen, resulting in excessive production of reactive oxygen species (ROS) $)^{3,4}$. Thus, rotenone administration has been used to elucidate the mechanisms underlying oxidative stress-induced neural degeneration following mitochondrial dysfunction ${ }^{5-14}$.

Chronic rotenone administration in rodents mimics the behavioral and pathological features observed in patients with Parkinson's disease, such as loss of nigrostriatal dopaminergic neurons, the formation of Lewy body-like filamentous protein inclusions in degenerated neurons, and impaired locomotor activity ${ }^{5,6,10-14}$. A previous study reported retinal impairments preceding the loss of dopaminergic neurons in the substantia nigra of rotenone-administered rats; thus, retinal change is an early marker of Parkinson's disease, at least in the rotenone-induced disease model ${ }^{15}$. Continuous intraperitoneal administration of rotenone results in elevated death of retinal ganglion cells (RGCs) and thinning of the swollen retina, probably owing to enhanced inflammation ${ }^{15}$. Furthermore, subcutaneous and intravitreal rotenone administration decreased the thickness of retinal layers, including the nerve layer, inner/outer nuclear layers, and inner plexiform layer, and fewer RGCs in rodents ${ }^{7,8,16,17}$. The putative mechanisms underlying rotenone-induced inner retinal degeneration have been described with an emphasis on neurons previously ${ }^{7}$.

\footnotetext{
${ }^{1}$ Department of Ophthalmology, Tohoku University Graduate School of Medicine, 1-1 Seiryo-machi, Aoba-ku, Sendai 980-8574, Japan. ${ }^{2}$ Department of Retinal Disease Control, Tohoku University Graduate School of Medicine, Sendai 980-8574, Japan. ${ }^{3}$ Collaborative Program of Ophthalmic Drug Discovery, Tohoku University Graduate School of Medicine, Sendai 980-8574, Japan. ${ }^{4}$ Department of Advanced Ophthalmic Medicine, Tohoku University Graduate School of Medicine, Sendai 980-8574, Japan. ${ }^{5}$ Department of Ophthalmic Imaging and Information Analytics, Tohoku University Graduate School of Medicine, Sendai 980-8574, Japan. ${ }^{6}$ These authors contributed equally: Hiroshi Tawarayama and Maki Inoue-Yanagimachi. ${ }^{\circledR}$ email: ntoru@oph.med.tohoku.ac.jp
} 
A

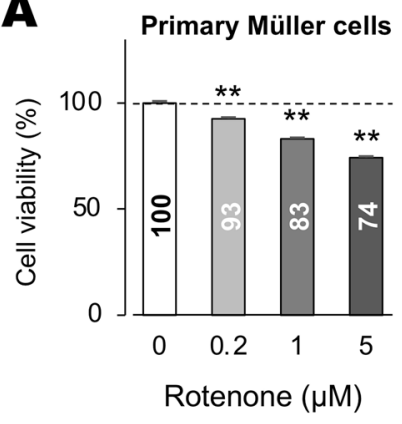

B

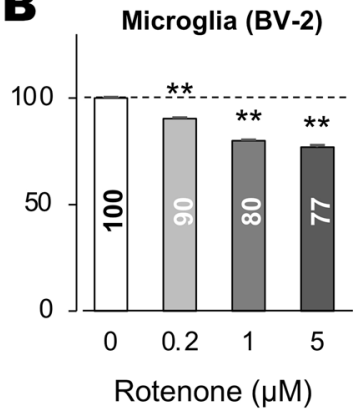

Figure 1. Viability of rotenone-treated Müller and BV-2 cells. (A, B) AlamarBlue cell viability assay of mouse primary Müller cells (A) and mouse brain-derived microglia BV-2 cells (B). Cell viability decreased in a dosedependent manner for rotenone. Error bars indicate standard deviation. ${ }^{* *} P<0.01$ versus rotenone (-) controls (Dunnett's test; $n=4$ ).

Glial cells, including Müller cells and microglia, play numerous roles, namely, metabolism, cell debris removal, trophic factor release, and incorporation of excessive neurotransmitters, to maintain functional stability of retinal cells in normal and pathological conditions ${ }^{18-21}$. Thus, dysfunction of Müller cells and microglia is often concomitant with the development of retinal degeneration diseases owing to the loss of neuronal supporting activities ${ }^{21-24}$. Previous studies suggested that glial cells modulated retinal survival and inflammation through altered secretion of neurotrophic factors and inflammatory cytokines in pathological conditions $\mathrm{s}^{25-27}$. Light irradiation, leading to photoreceptor cell death, upregulates growth factors, including fibroblast growth factor 2 (FGF2), nerve growth factor (NGF), glial cell line-derived neurotrophic factor (GDNF), and ciliary neurotrophic factor (CNTF) in microglia ${ }^{25}$. Moreover, the secretion of light-irradiated microglia upregulated brain-derived neurotrophic factor (BDNF), whereas it downregulated FGF2 in Müller cells ${ }^{25}$. Thus, glial secretion may modulate retinal cell survival during neural degeneration.

Since mitochondrial dysfunction-mediated oxidative stress contributes to the pathogenesis of retinal diseases, such as Leber's hereditary optic neuropathy and glaucoma, rotenone administration provides an excellent model to study the mechanisms underlying oxidative stress-related retinal degeneration ${ }^{9,28-30}$. Additionally, the role of glial cells in rotenone-induced retinal degeneration remains elusive. Thus, to further characterize the rotenoneinduced retinal degeneration model, we examined the roles of glia and their secretion in our study.

\section{Results}

Dose-dependent effects of rotenone on the survival of Müller and microglial cells. Mouse primary Müller cells and mouse BV-2 cells, instead of primary retinal microglia, were used to investigate the roles of glial cell secretion in rotenone-induced retinal degeneration since the number of retina-derived primary microglia was expected to be small to perform all our experiments. Past studies and our findings indicated that both primary microglia and BV-2 cells possessed the common properties to produce the same kinds of cytokines and growth factors (see the text below) ${ }^{25,31-33}$.

We first monitored the dose-dependent effects of rotenone on survival of Müller and BV-2 cells using the alamarBlue assay; rotenone significantly decreased the viability of these cells in a dose-dependent manner (Fig. 1A,B).

Rotenone-induced expression changes of growth factors and inflammatory cytokines in Müller and BV-2 cells. We then examined expression changes of growth factors, including Ngf, Bdnf, Ntf3, Ntf5, $G d n f$, Cntf, and Fgf2 genes, and inflammatory cytokines, including interleukin (Il) $1 \beta$, Il8, C-C motif chemokine ligand $2(\mathrm{Ccl} 2)$, and tumor necrosis factor-alpha (Tnf- $\alpha)$ genes, in Müller and BV-2 cells treated with various concentrations of rotenone using qRT-PCR.

Rotenone treatment increased the expression of Fgf2, Cntf, Gdnf, and Il6 genes, whereas the expression of $B d n f$ and $C c l 2$ genes decreased in Müller cells (Fig. 2A). No significant change was observed in the expression level of the $N g f$ gene (Fig. 2A). In contrast, rotenone treatment significantly increased the expression of $N t f 5$, Fgf2, Cntf, Tnf- $\alpha, C c l 2, I l 1 \beta, I l 6$, and $I l 8$ genes in BV-2 cells (Fig. 2B). The expression level of Ill $\beta$, Tnf- $\alpha$, and $N t f 3$ genes in Müller cells and the expression of $N g f, B d n f$, Gdnf, and Ntf3 genes in BV-2 cells were lower than the detection limit (data not shown).

Expression changes of growth factors and inflammatory cytokines in Müller and BV-2 cells stimulated with rotenone-treated cell conditioned media. We then examined the effects of glial secretion of rotenone-treated cells on gene expression of growth factors and inflammatory cytokines in Müller and BV-2 cells. Müller and BV-2 cells were cultured in media containing various concentrations of rotenone for $24 \mathrm{~h}$, followed by culturing in fresh media for 24 more hours after washing rotenone-treated cells. Naïve Müller and BV-2 cells were cultured in conditioned media prepared from rotenone-treated BV-2 and Müller cells for $6 \mathrm{~h}$, respectively, and the expression changes were analyzed using qRT-PCR. 

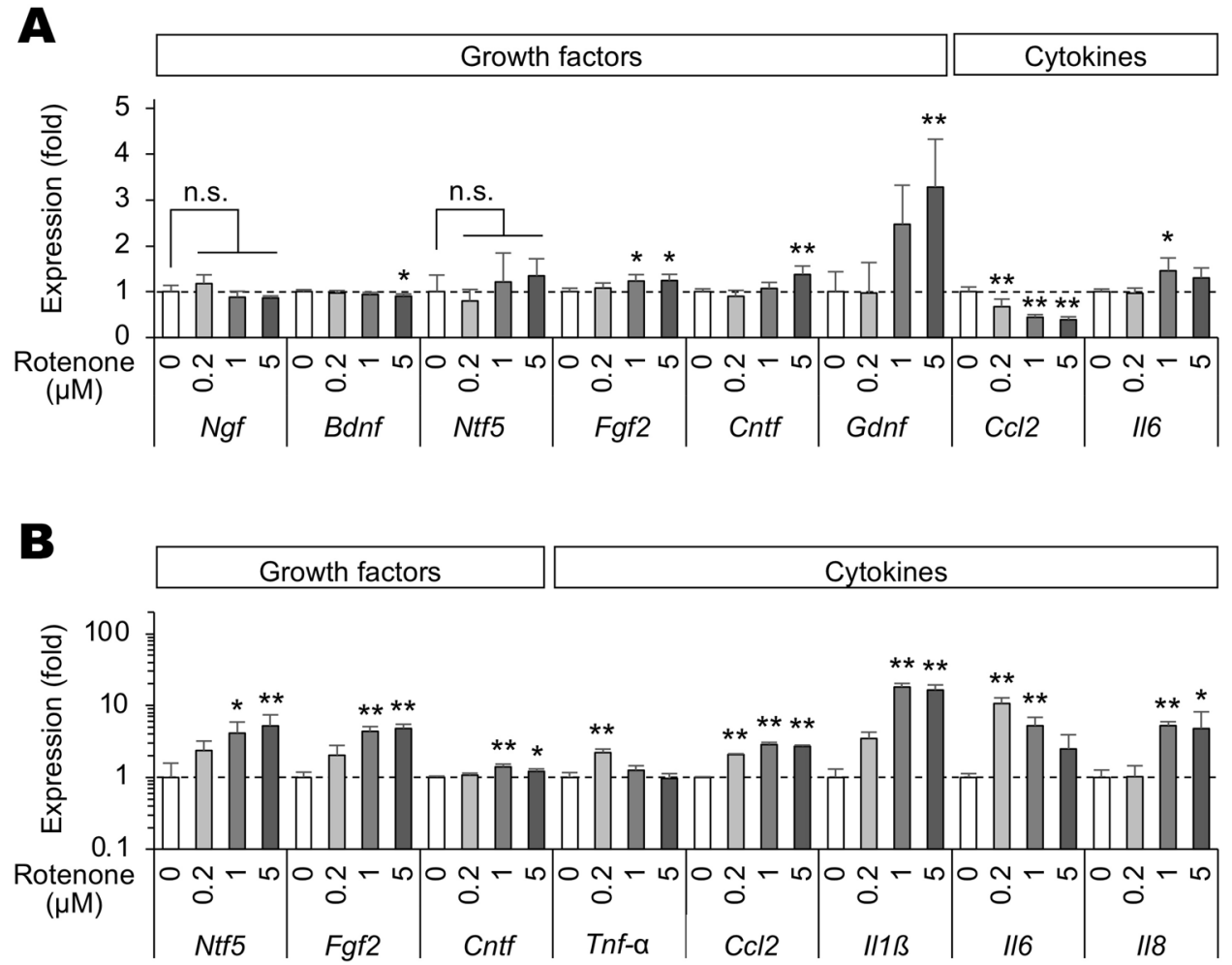

Figure 2. Rotenone-induced gene expression changes of growth factors and inflammatory cytokines in Müller and BV-2 cells. (A, B) Expression of $N g f, B d n f, N t f 5, F g f 2$, Cntf, Gdnf, Ccl2, and Il6 genes in Müller cells (A) and expression of Ntf5, Fgf2, Cntf, Tnf- $\alpha, C c l 2, I l 1 \beta, I l 6$, and $I l 8$ genes in BV-2 cells (B) treated with various concentrations of rotenone for $24 \mathrm{~h}$. Expression levels were normalized against those of the Gapdh gene and were shown as values relative to those of each rotenone $(-)$ control. Error bars indicate standard deviation. ${ }^{\star} P<0.05,{ }^{* *} P<0.01$ versus rotenone $(-)$ control (Dunnett's test; $\left.n=4\right)$ ). n.s.: not significant.

Conditioned media prepared from rotenone-stimulated BV-2 cells increased the expression level of $B d n f$ and Fgf2 genes in Müller cells, whereas the expression level of Gdnf and Il6 genes decreased (Fig. 3A). It did not affect the expression level of $\mathrm{Ngf}, \mathrm{Ntf5}, \mathrm{Cntf}$, and $\mathrm{Ccl} 2$ genes (Fig. 3A). In contrast, conditioned media prepared from rotenone-stimulated Müller cells increased the expression level of Ntf5, Fgf2, Cntf, Tnf- $\alpha$, Ccl2, Ill $\beta$, AND Il6 genes in BV-2 cells but did not affect Il8 expression level (Fig. 3B).

Effects of rotenone-induced glial cell secretion on oxidative stress-induced death of dissociated retinal cells. We then examined the effects of secreted molecules from rotenone-stimulated cells and cells stimulated with rotenone-treated cell conditioned media on oxidative stress-induced death in dissociated retinal cells. Conditioned media prepared in different glial cell combinations presented in Fig. 4A (Media 1-4) were used for the experiment (see Materials and methods for details). Dissociated mouse retinas were cultured in media 1 (rotenone-treated BV-2 cell-conditioned media), Media 2 (rotenone-treated Müller cell-conditioned media), Media 3 (Media 1-treated Müller cell-conditioned media), and Media 4 (Media 2-treated BV-2 cellconditioned media). Cell viability was then determined using the alamarBlue assay. Media 1 and 4 significantly increased cell viability in dissociated retinal cell cultures (Fig. 4B,E), whereas Media 2 decreased cell viability (Fig. 4C). In contrast, Media 3 did not affect cell viability (Fig. 4D).

Intravitreal Ntf5 administration inhibited rotenone-induced retinal cell death. Media 1 and 4 increased the survival of dissociated retinal cells (Fig. 4), and both stimulated Ntf5 expression in BV-2 cells (Figs. 2B, 3B). Thus, to examine the in vivo effects of NTF5 on the rotenone-induced loss of retinal cells, rats were intravitreally administered with rotenone $(30 \mathrm{nmol})$ and NTF5 recombinant proteins $(0.15 \mathrm{or} 1.5 \mu \mathrm{g})$, and RBPMS immunohistochemistry and HE staining were performed on retinal sections seven days later (Fig. 5A). Intravitreal rotenone administration resulted in fewer RBPMS ${ }^{+}$RGCs and thinner INL (Fig. 5B), which were consistent with previous findings ${ }^{7}$. However, NTF5 recombinant proteins attenuated rotenone-induced retinal impairment in a dose-dependent manner (Fig. 5C,D). 

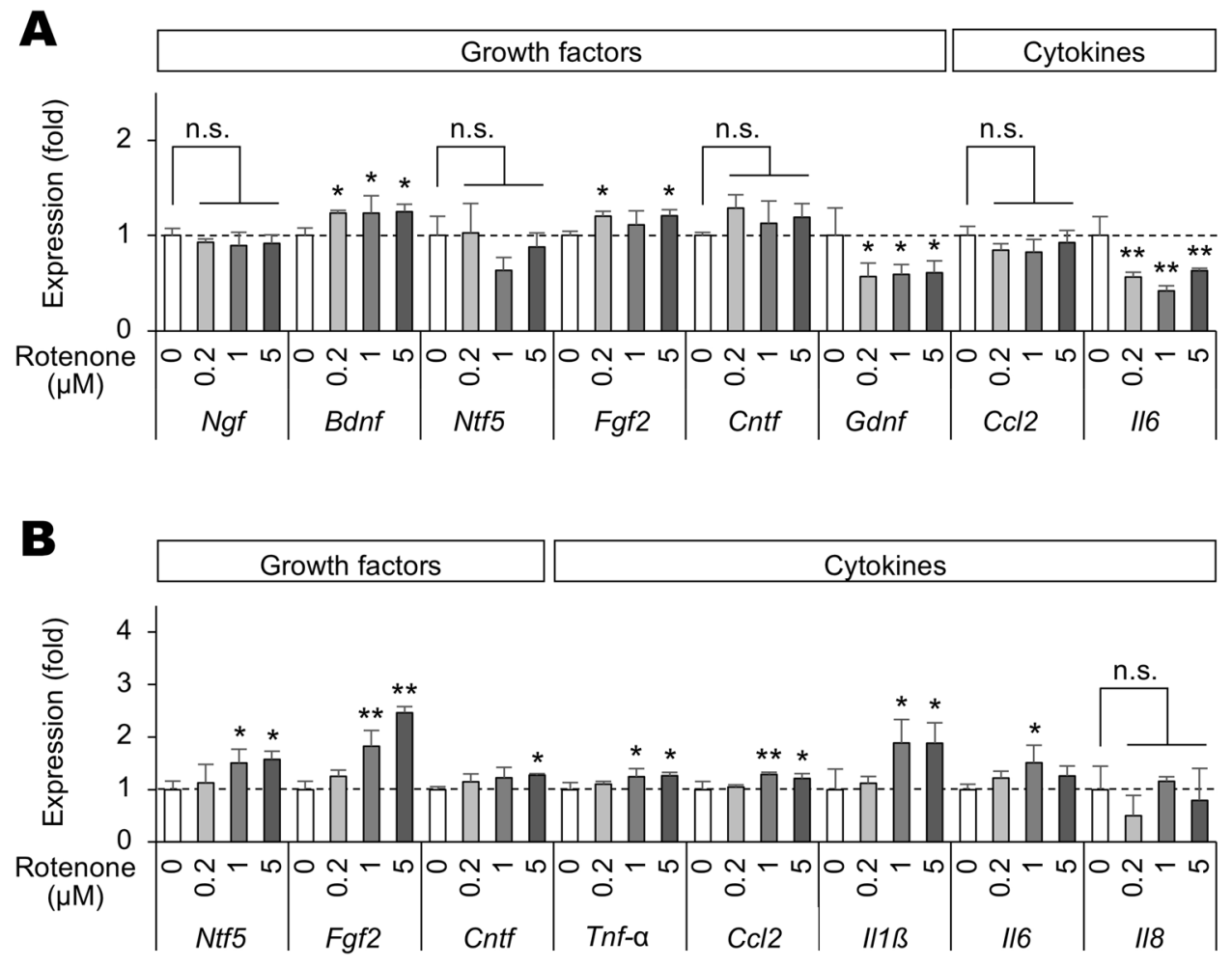

Figure 3. Rotenone-treated cell-conditioned media-induced gene expression changes of growth factors and inflammatory cytokines in Müller and BV-2 cells. (A, B) Expression of Ngf, Bdnf, Ntf5, Fgf2, Cntf, Gdnf, Ccl2, and $I l 6$ genes in Müller cells treated with conditioned media of BV-2 cells (A) and expression of Ntf5, Fgf2, Cntf, Tnf- $\alpha, C c l 2, I l 1 \beta, I l 6$, and Il8 in BV-2 cells treated with conditioned media of Müller cells (B) and stimulated with various concentrations of rotenone for $6 \mathrm{~h}$. Expression levels were normalized against those of the GAPDH gene and were shown as values relative to those of each rotenone $(-)$ control. Error bars indicate standard deviation. ${ }^{\star} P<0.05,{ }^{* *} P<0.01$ versus rotenone $(-)$ control (Dunnett's test; $n=4$ ). n.s.: not significant.

\section{Discussion}

The present study indicated that administration of mitochondrial complex I inhibitor rotenone or rotenonestimulated cell-conditioned media altered gene expression of these molecules in glial cells, as summarized in Fig. 6A. This altered expression would potentially modulate retinal cell survival (Fig. 6B). Consistent with the present findings, it has been shown that neurotoxic stimuli and the following glial cell secretions activated the production of not only proinflammatory cytokines but also growth factors in Müller and microglial cells ${ }^{25,34-39}$. Some studies reported the mechanisms underlying the glial secretion-mediated alteration of growth factor gene expression in other glia: Microglia-derived NGF downregulates the Fgf2 expression in cultured Müller cells via activation of a common neurotrophin receptor p75 under oxidative stress conditions ${ }^{25,40}$. This leads to enhanced cell death of photoreceptors due to the decrease of the FGF2-mediated neuroprotective activity ${ }^{25,40}$. Similarly, dysregulated expression of growth factors and inflammatory cytokines in glial cells and its secondary effects via the glial secretions could modulate cell survival and death in the rotenone-induced retinal degeneration.

Neuroprotective effects of NTF5 have been reported previously ${ }^{41-44}$. Retinal cells displayed higher susceptibility to ischemic injuries in Ntf5-deficient animals than wild-type animals ${ }^{43}$. RGCs were more resistant to cell death following optic nerve crush in alpha-lipoic acid-administered rats where $N t f 5$ expression was upregulated in $\mathrm{RGCs}^{44}$. Furthermore, NTF5 could prevent naturally occurring retinal cell death during development ${ }^{42,43}$. Since our findings indicated that (1) Ntf5 expression was upregulated in BV-2 cells in response to rotenone and rotenone-stimulated Müller cell-conditioned media, (2) conditioned media obtained from these BV-2 cell cultures (Media 1 and 4, Fig. 4A) promoted the viability of dissociated retinal cells, and (3) intravitreal Ntf5 administration attenuated rotenone-induced retinal degeneration, we concluded that microglia exerted retinal cell survival-promoting activity by upregulating NTF5 expression.

It is known that not only NTF5 but also BDNF preferentially utilize the tropomyosin-related kinase (Trk) $B$ receptor, a member of the neurotrophic tyrosine kinase receptor family, as a signal transducer, suggesting that BDNF plays identical roles to NTF5 in regulating retinal cell survival ${ }^{45-47}$. Supporting this, past studies reported the neuroprotective effect of BDNF on retinal cells in animal glaucoma models and hypoxia- and glucose deprivation-induced injury models ${ }^{48-50}$. However, in our study, media prepared from Müller cells treated with rotenone-stimulated BV-2 cell-conditioned media (Media 3, Fig. 4A) had no effects on the viability of dissociated retinal cell cultures, although it induced Bdnf upregulation in Müller cells (Fig. 3A). NTF5 and BDNF had similar but distinguishable roles in synaptic transmission, neuronal survival, and growth in a context-dependent manner ${ }^{51-54}$. Additionally, it has been reported that NTF5 and BDNF display different binding properties to 
$\mathbf{A}$
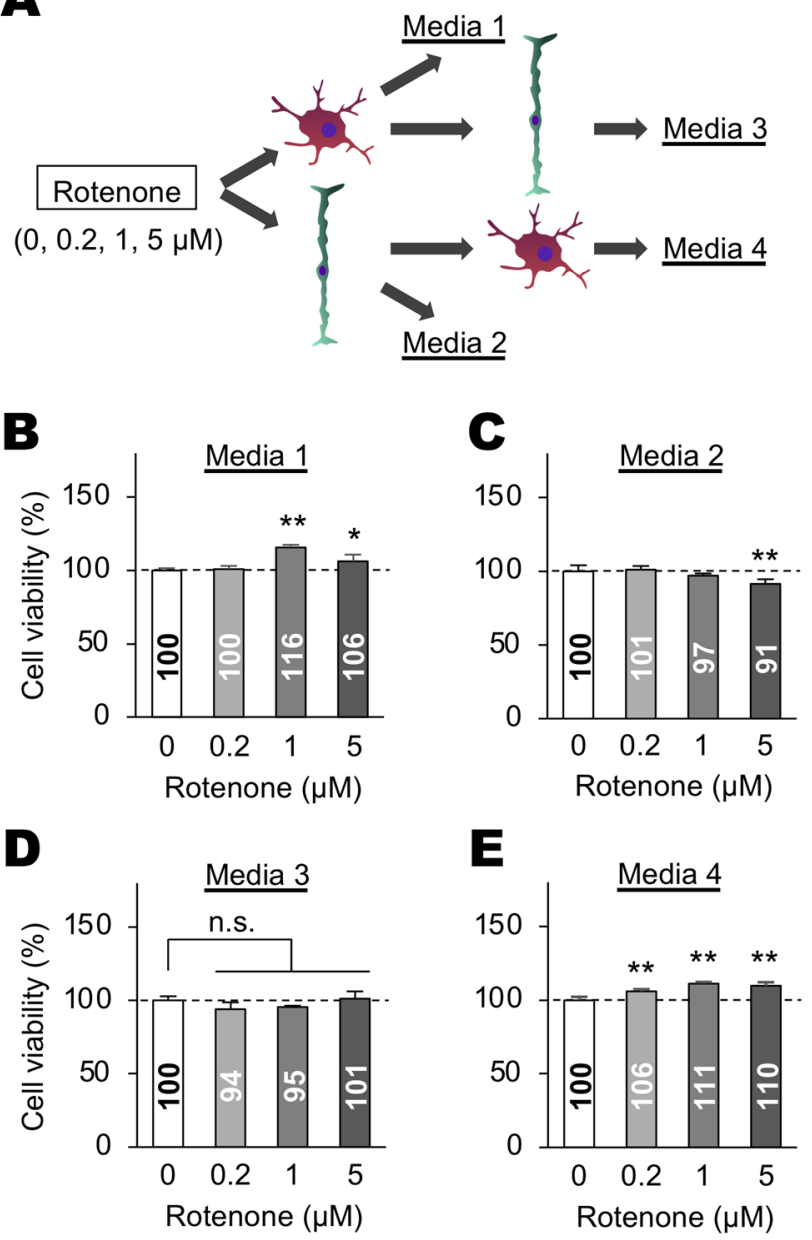

Figure 4. Effects of conditioned media of rotenone-treated Müller and BV-2 cells on survival of dissociated retinal cells. (A) Schematic for the preparation of conditioned media of rotenone-treated Müller and BV-2 cells. BV-2 and Müller cells were treated with various concentrations of rotenone for $24 \mathrm{~h}$ and were then cultured with fresh media for $24 \mathrm{~h}$ more. The conditioned media of BV-2 and Müller cells were collected (Media 1 and 2, respectively). Naïve Müller and BV-2 cells were cultured with Media 1 and 2 for $24 \mathrm{~h}$ and were then collected (Media 3 and 4, respectively). (B-E) Cell viability assay on mouse dissociated retinal cells cultured with Media 1-4 for $24 \mathrm{~h}$, respectively. Viability was shown as values relative to that of rotenone $(-)$ control. Error bars indicate standard deviation. ${ }^{\star} P<0.05$ and ${ }^{*} P<0.01$ versus rotenone $(-)$ control (Dunnett's test; $n=4$ ). n.s.: not significant.

the TrkB receptor ${ }^{52,55}$. Thus, the differences in molecular properties between NTF5 and BDNF might explain the reason conditioned media rich in NTF5 but not rich in BDNF promoted retinal cell survival in our study. Alternatively, the increasing rate of $B d n f$ upregulation in Müller cells, stimulated with BV-2-conditioned media (Media 3), was too less to promote the viability of dissociated retinal cells, or Müller cells produce toxic molecules to decrease retinal cell survival (Fig. 4C), which antagonize the cell survival-promoting activity of BDNF.

Our in vivo study demonstrated that intravitreal administration of NTF5 attenuated the rotenone-induced loss of RGCs and INL cells in a dose-dependent manner. Given that the TrkB receptor is expressed in RGCs and amacrine cells in the ganglion cell and inner nuclear layers, NTF5 could rescue rotenone-induced cell death of these cells by its direct effect through TrkB-mediated signaling ${ }^{56,57}$. Moreover, NTF5 might be implicated in the prevention of rotenone-induced retinal cell death in an indirect manner, i.e., through the activation of Müller cells expressing TrkB and downregulation of other growth factors secreted from glia similar to FGF2-mediated BDNF downregulation described above ${ }^{25,40,58}$. Both the direct and indirect effects of microglia-derived NTF5 could play pivotal roles in the modulation of survival in various types of retinal cells despite the TrkB expression. Although we have not focused on the impact of astrocytes on rotenone-induced retinal degeneration in this study, astrocytes also contribute to the promotion of retinal cell survival; the neuroprotective effects of astrocytes in injured retinal tissues has been demonstrated by previous studies ${ }^{59}$.

Our study indicated that treatment with rotenone or rotenone-stimulated Müller cell-conditioned media significantly increased the expression level of $I l 1 \beta$ and $I l 6$ in BV-2 cells. These cytokines exert overlapping and synergistic activities to stimulate the production of other inflammatory cytokines ${ }^{60-63}$. Thus, IL1ß and IL6 would exacerbate an inflammation state, leading to excessive neural cell death. In contrast, some studies have 
A

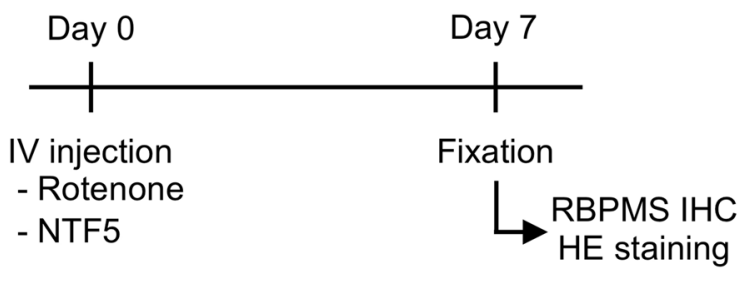

B

\section{Rotenone} $(30 \mathrm{nmol})$

NTF5 $(\mu \mathrm{g})$

0

0

0.15

1.5

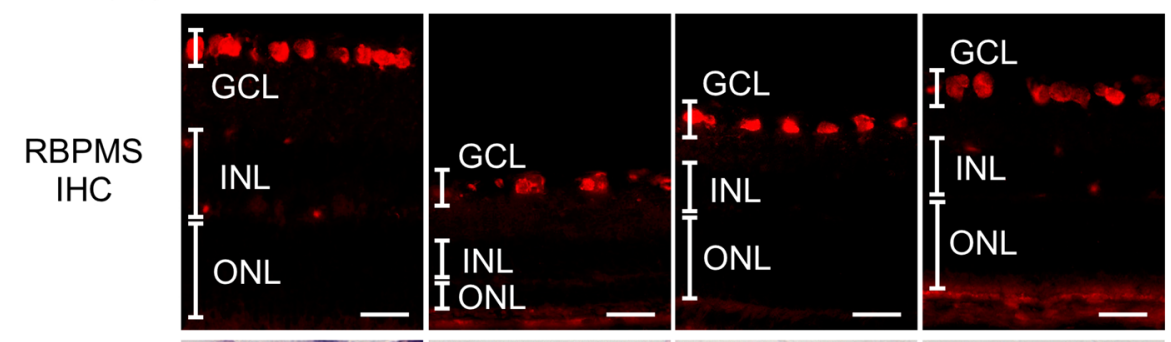

HE staining
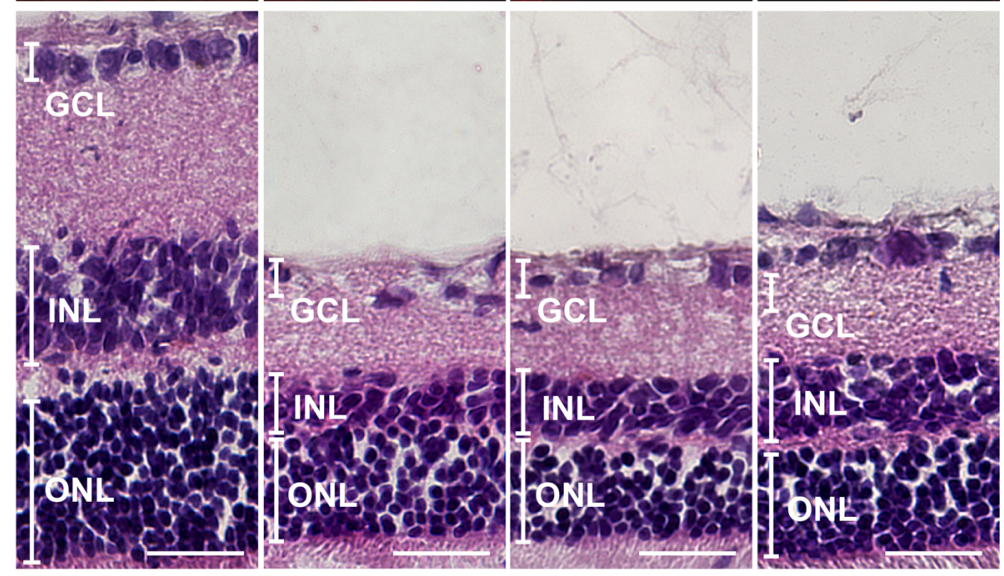

C

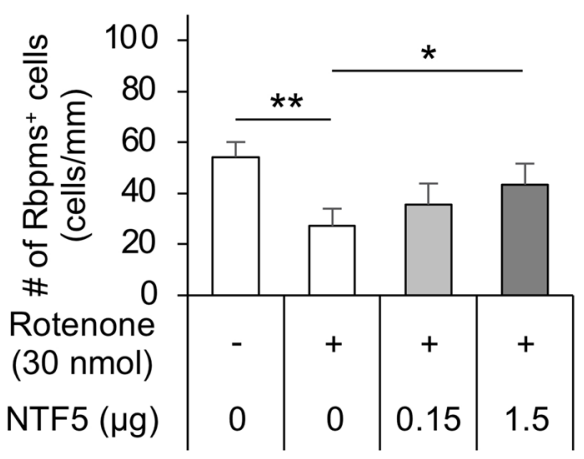

D

Figure 5. Effects of intravitreal administration of NTF5 proteins on rotenone-induced retinal degeneration in rats. (A) Schematic of the experimental procedure. (B) RBPMS immunohistochemistry (IHC) and hematoxylin/ eosin (HE) staining of retinal sections prepared from animals intravitreally treated with rotenone and NTF5 recombinant proteins. (C, D) Quantification of the number of RBPMS ${ }^{+} \mathrm{RGCs}(\mathbf{C})$ and thickness of the INL in rotenone- and NTF5-administrated rats. RBPMS, RNA-binding protein with multiple splicing; RGC, retinal ganglion cell. Scale bars: $50 \mu \mathrm{m}$. 
A

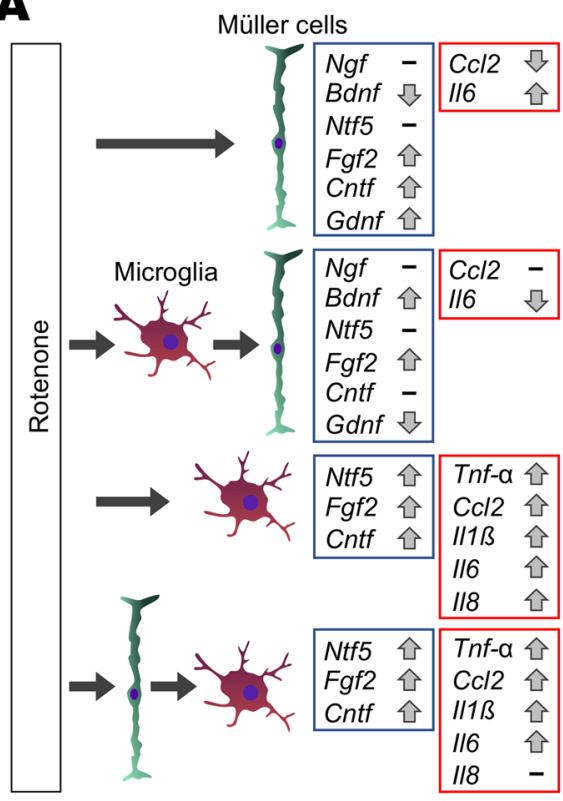

B

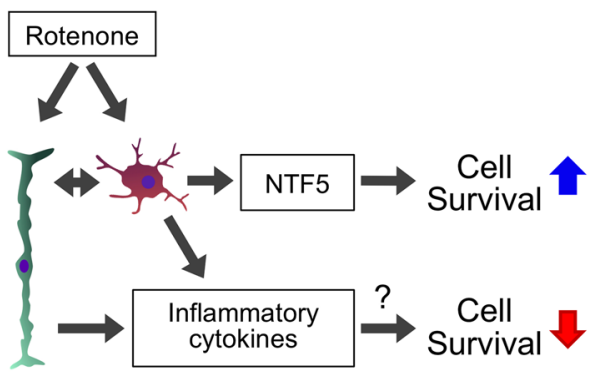

Figure 6. Schematic diagram explaining glial roles in rotenone-induced retinal degeneration. (A) Summary of expression changes in growth factors and inflammatory cytokines in Müller and BV-2 cells stimulated with rotenone or rotenone-stimulated cell-conditioned media. Gene expression changes of growth factors and inflammatory cytokines were shown in the boxes colored blue and red, respectively. (B) Positive and negative effects of rotenone-stimulated glial cells on retinal survival. Expression of inflammatory cytokines is upregulated in rotenone-treated Müller and microglial cells. These would result in a decreased survival of retinal cells owing to excessive inflammatory responses. In contrast, microglia activate to produce NTF5 in response to rotenone or secretion of rotenone-treated Müller cells, which leads to increased survival in retinal cells owing to its neuroprotective activity.

demonstrated the positive effects of IL1ß and IL6 on retinal cell survival ${ }^{64,65}$. Intravitreal administration of IL1ß or IL6 resulted in microglial activation and neural survival promotion in N-methyl-D-aspartate (NMDA)-treated retinas $^{64,65}$. Deficiency of IL1ß receptor (IL1R1) and microglial ablation diminished the survival-promoting effects of these cytokines ${ }^{64,65}$. However, administration of NMDA prior to IL6 administration resulted in exacerbation of NMDA-induced retinal degeneration ${ }^{64}$. These findings suggested that proinflammatory cytokines such as IL1ß and IL6 secreted from activated microglia exerted positive and negative effects on retinal cell survival in normal and pathological conditions, respectively. Thus, increased expression of proinflammatory cytokines may result in enhanced neural cell death in rotenone-treated retinas.

In summary, we investigated the potential roles of glial cells and their secretion in rotenone-induced retinal degeneration reflecting the pathology of mitochondrial oxidative stress-induced retinal diseases in this study ${ }^{8,9,16}$. The obtained results suggested that the microglia-derived NTF5 prevents rotenone-induced retinal cell death. Thus, adeno-associated virus-based constitutive expression of exogenous NTF5 in the microglia or intravitreal administration of exogenous NTF5 would be effective in treating oxidative stress-associated retinal degeneration diseases.

\section{Methods}

Animals. C57BL/6 male and female mice (8-10 weeks old) and Sprague-Dawley (SD) male rats (8-10 weeks old) were purchased from SLC (Shizuoka, Japan) and maintained at animal facilities in Tohoku University Graduate School of Medicine (Sendai, Japan) under a 12-h light/dark cycle. Mice were mated to obtain pups for primary Müller cell preparation. Male mice were used for retinal dissociation culture experiments. All experimental procedures conformed to "Regulations for Animal Experiments and Related Activities at Tohoku University" and were reviewed by the Institutional Laboratory Animal Care and Use Committee of Tohoku University, and finally approved by the President of University. This study was performed in compliance with the ARRIVE guidelines.

Preparation of mouse primary Müller cells. Preparation and culture of Müller cells have been described previously ${ }^{25,66}$. Briefly, eyes dissected from postnatal day (P)5 to P8 mouse pups were incubated in Dulbecco's modified Eagle medium (DMEM; Thermo Fisher Scientific, Waltham, MA, USA) containing 10\% fetal bovine serum (FBS; Thermo Fisher Scientific) at room temperature overnight. After washing with Dulbecco's phosphate buffered saline, eyes were treated with $0.25 \%$ trypsin solution for $15 \mathrm{~min}$. Retinas were isolated from eyes using sharp forceps and were broken into small pieces by pipetting several times. Prepared retinal explants were cultured in DMEM containing $10 \%$ FBS (DMEM10) in a $\mathrm{CO}_{2}$ incubator at $37^{\circ} \mathrm{C}$. Growing Müller cells out of the 


\begin{tabular}{|l|l|l|l|}
\hline Species & Genes & Supplier & Assay ID \\
\hline mouse & Gapdh & Integrated DNA Technologies & Mm.PT.39a.1 \\
\hline mouse & $\mathrm{Ngf}$ & Integrated DNA Technologies & Mm.PT.58.14181538 \\
\hline mouse & Bdnf & Integrated DNA Technologies & Mm.PT.58.8157970 \\
\hline mouse & Ntf5 & Integrated DNA Technologies & Mm.PT.58.11243625 \\
\hline mouse & Fgf2 & Integrated DNA Technologies & Mm.PT.56a.5129235 \\
\hline mouse & Cntf & Integrated DNA Technologies & Mm.PT.58.32700675.g \\
\hline mouse & Gdnf & Integrated DNA Technologies & Mm.PT.58.6003912 \\
\hline mouse & Tnf- $\alpha$ & Integrated DNA Technologies & Mm.PT.58.12575861 \\
\hline mouse & Ccl2 & Integrated DNA Technologies & Mm.PT.58.42151692 \\
\hline mouse & $I l-1 \beta$ & Integrated DNA Technologies & Mm.PT.58.41616450 \\
\hline mouse & $I l-6$ & Integrated DNA Technologies & Mm.PT.58.10005566 \\
\hline mouse & $I l-8$ & Integrated DNA Technologies & Mm.PT.58.9981538 \\
\hline
\end{tabular}

Table 1. List of the primer and probe mixtures used for quantitative qRT-PCR.

explants were sub-cultured upon reaching $80 \%$ confluency using a trypsin solution. Primary Müller cells with high passage numbers displayed drastic growth inhibition and morphological change. Thus, Müller cells passaged less than 6 times were used for the in vitro experiments. Müller cell cultures are shown in the supplementary figure. We identified Müller cells based on their characteristic shape that helped us distinguish them from astrocytes ${ }^{67}$. Nearly all of the cultured cells displayed the morphological features of Müller cells.

Conditioned media preparation of rotenone-treated Müller cells and microglia. Mouse primary Müller cells, prepared as described previously, and mouse brain-derived microglia (BV-2) were maintained in DMEM10. Müller and BV-2 cells were seeded at $3.0 \times 10^{5}$ and $6.5 \times 10^{5}$ cells in $60 \mathrm{~mm}$ dishes, respectively, and cultured in media containing various concentrations of rotenone (Tokyo chemical industry, Tokyo, Japan) for $24 \mathrm{~h}$. Cells were washed with DMEM10 twice to remove rotenone and were then cultured in fresh media for an additional $24 \mathrm{~h}$. Culture media were collected and passed through a $0.22 \mu \mathrm{m}$ filter (Merck Millipore, Burlington, MA, USA). To obtain double-conditioned media, Müller and BV-2 cells $\left(1.4 \times 10^{5}\right.$ and $3.0 \times 10^{5}$ cells, respectively) were cultured with rotenone-stimulated BV-2 and Müller cell-conditioned media ( $3 \mathrm{~mL}$ ) in $35 \mathrm{~mm}$ dishes for $24 \mathrm{~h}$, respectively.

Quantitative reverse transcription-polymerase chain reaction (qRT-PCR). Primary Müller cells and BV-2 cells were seeded at $0.5 \times 10^{4}$ and $5 \times 10^{4}$ cells/well in 96 -well cell culture plates, respectively, and were treated with various concentrations of rotenone for $24 \mathrm{~h}$. In some cases, these cells were treated with conditioned media instead of rotenone containing media for $6 \mathrm{~h}$. After cell lysis in each well of the 96-well cell culture plates, RNA was reverse transcribed into cDNA using the SuperPrepII cell lysis \& RT kit (Toyobo, Osaka, Japan) according to the manufacturer's instruction. qRT-PCR was performed in a 7500 fast real-time PCR system (Thermo Fisher Scientific) using TaqMan fast universal PCR master mix (Thermo Fisher Scientific) and a mixture of predesigned TaqMan primers and probes [Thermo Fisher Scientific or Integrated DNA Technologies (Coralville, IA, USA)] (see Table 1). Gene expression was examined in 4 individual wells of the 96-well plates per each treatment $(n=4)$, and the average and standard deviation were subsequently calculated. The gene expression level is shown as a percentage of rotenone-untreated controls.

Retinal dissociation culture. Retinal dissociation cultures were performed as described previously ${ }^{68}$. Briefly, 6 retinas were dissected from 3 male mice (8-10 weeks old) and dissociated into single cells using the neural tissue dissociation kit (Miltenyi Biotec, Bergisch Gladbach, Germany). Dissociated retinal cells were mixed and resuspended in Neurobasal-A medium (Thermo Fisher Scientific) containing B-27 supplement without antioxidants (Thermo Fisher Scientific). Cells were seeded at $1 \times 10^{5}$ cells/well $(50 \mu \mathrm{L})$ in 96 -well cell culture plates. One hour after seeding, the same volume of Müller and/or BV-2 cell-conditioned media was added to retinal cell mixtures and cultured for $24 \mathrm{~h}$.

Cell viability assay. Primary Müller cells, BV-2 cells, and dissociated retinal cells were seeded at $0.5 \times 10^{4}$, $5 \times 10^{4}$, and $1 \times 10^{5}$ cells/well in 96-well cell culture plates, respectively, and were incubated in DMEM10 containing various concentrations of rotenone or Müller and/or BV-2 cell-conditioned media for $24 \mathrm{~h}$. Culture media were removed, and cells were then incubated in DMEM10 containing $10 \%$ of the alamarBlue cell viability reagent (Thermo Fisher Scientific) for $3 \mathrm{~h}$. In the case of retinal dissociated cells, alamarBlue was added to cultures one hour after seeding. Fluorescence intensity was measured at $590 \mathrm{~nm}$ (excitation: $560 \mathrm{~nm}$ ) using a SpectraMax M2e microplate reader (Molecular Devices, San Jose, CA, USA). The alamarBlue signals were examined in 4 individual wells of 96-well plates per each treatment $(n=4)$, and the average and standard deviation were subsequently calculated. The viability is shown as a percentage of the rotenone-untreated controls. The same experiments using the cell cultures prepared from different individuals were performed twice to confirm the obtained results. 
Intravitreal administration. SD rats were anesthetized using intraperitoneal injection of $8 \mathrm{mg} / \mathrm{kg}$ xylazine (Bayer Yakuhin, Osaka, Japan) and $80 \mathrm{mg} / \mathrm{kg}$ ketamine (Daiichi Sankyo, Tokyo Japan). A mixture of $2.5 \mu \mathrm{L}$ of rotenone dissolved in dimethyl sulfoxide $(30 \mathrm{nmol})$ and/or the same volume of neurotrophin (NTF) 5 recombinant proteins $\left(0.15\right.$ or $1.5 \mu \mathrm{g}$ ) dissolved in $\mathrm{Ca}^{2+}$ and $\mathrm{Mg}^{2+}$-free Dulbecco's phosphate buffered saline (DPBS; $10 \mathrm{mM}, \mathrm{pH}$ 7.4) were administered intravitreally using a micro syringe with a 32-G sharp needle (Ito corporation, Shizuoka, Japan). DPBS was used as an injection control.

Histology. Eyes were dissected from SD rats seven days after intravitreal injection of rotenone alone or together with Ntf5 recombinant proteins and were fixed with $4 \%$ paraformaldehyde in PBS overnight at $4{ }^{\circ} \mathrm{C}$. Fixed eyes were embedded in optimal cutting temperature compound (Sakura Finetek Japan, Tokyo, Japan) and were cut into $10 \mu \mathrm{m}$-thick sections using a cryostat CM3050S (Leica Biosystems, Wetzlar, Germany). Immunostaining of retinal sections was performed as reported before ${ }^{69}$. Briefly, retinal sections were treated with blocking solution ( $10 \%$ normal donkey serum in PBS containing $0.01 \%$ Tween 20 ), followed by treatment with rabbit primary antibody (Abcam, Cambridge, UK; 1:500 dilutions with blocking solution) for RNA-binding protein with multiple splicing (RBPMS), which is a selective marker for retinal ganglion cells ${ }^{70}$. After extensive washing, retinal sections were incubated with Cy3-conjugated anti-rabbit IgG antibody (Jackson ImmunoResearch, West Grove, PA, USA; 1:500 dilutions with blocking solution) and 4',6-diamidino-2-phenylindole (DAPI; Dojindo, Kumamoto, Japan). To visualize the inner nuclear layer (INL) cells, retinal sections were stained with hematoxylin and eosin (HE; Muto Pure Chemicals, Tokyo, Japan).

Quantification of the retinal ganglion cell number and the INL thickness. Photos were acquired using a fluorescent microscope BZ-X810 (Keyence, Osaka, Japan) with a $20 \times$ objective lens on microscopic fields $200 \mu \mathrm{m}$ far from the optic nerve head of at least three retinal sections stained with RBPMS antibodies or HE per eye. The number of RBPMS ${ }^{+}$RGCs and INL thickness in each image were quantified using ImageJ software (National Institute of Health, Bethesda, MD, USA). The average was calculated from five individual eyes per each experimental group.

Statistical analyses. Quantitative data were analyzed using the Tukey-Kramer and Dunnett's tests with JMP Pro 14 software (SAS Institute, Cary, NC, USA). A $P$-value of $<0.05$ was considered statistically significant.

\section{Data availability}

The datasets used and analyzed in the current study are available from the corresponding author upon request.

Received: 25 February 2021; Accepted: 12 May 2021

Published online: 27 May 2021

\section{References}

1. Soloway, S. B. Naturally occurring insecticides. Environ. Health Perspect. 14, 109-117 (1976).

2. Biggs, D. R., Nakamura, H., Kearney, E. B., Rocca, E. \& Singer, T. P. Studies on the respiratory chain-linked reduced nicotinamide adenine dinucleotide dehydrogenase. Arch. Biochem. Biophys. 137, 12-29 (1970).

3. Radad, K., Rausch, W. D. \& Gille, G. Rotenone induces cell death in primary dopaminergic culture by increasing ROS production and inhibiting mitochondrial respiration. Neurochem. Int. https://doi.org/10.1016/j.neuint.2006.02.003 (2006).

4. Sherer, T. B. et al. Mechanism of toxicity in rotenone models of Parkinson's disease. J. Neurosci. 23, 10756-10764 (2003).

5. Betarbet, R. et al. Chronic systemic pesticide exposure reproduces features of Parkinson's disease. Nat. Neurosci. 3, 1301-1306 (2000).

6. Sherer, T. B., Kim, J. H., Betarbet, R. \& Greenamyre, J. T. Subcutaneous rotenone exposure causes highly selective dopaminergic degeneration and $\alpha$-synuclein aggregation. Exp. Neurol. https://doi.org/10.1006/exnr.2002.8072 (2003).

7. Sasaoka, M., Ota, T. \& Kageyama, M. Rotenone-induced inner retinal degeneration via presynaptic activation of voltage-dependent sodium and L-type calcium channels in rats. Sci. Rep. 10, 1-16 (2020).

8. Zhang, X., Jones, D. \& Gonzalez-Lima, F. Mouse model of optic neuropathy caused by mitochondrial complex I dysfunction. Neurosci. Lett. https://doi.org/10.1016/S0304-3940(02)00327-0 (2002).

9. Zhang, L. et al. Long-term evaluation of Leber's hereditary optic neuropathy-like symptoms in rotenone administered rats. Neurosci. Lett. https://doi.org/10.1016/j.neulet.2014.12.004 (2015).

10. Fleming, S. M. et al. Behavioral and immunohistochemical effects of chronic intravenous and subcutaneous infusions of varying doses of rotenone. Exp. Neurol. https://doi.org/10.1016/j.expneurol.2004.01.023 (2004).

11. Cannon, J. R. et al. A highly reproducible rotenone model of Parkinson's disease. Neurobiol. Dis. 34, 279-290 (2009).

12. Pan-Montojo, F. et al. Progression of Parkinson's disease pathology is reproduced by intragastric administration of rotenone in mice. PLoS ONE 5, e8762 (2010).

13. Alam, M., Mayerhofer, A. \& Schmidt, W. J. The neurobehavioral changes induced by bilateral rotenone lesion in medial forebrain bundle of rats are reversed by L-DOPA. Behav. Brain Res. https://doi.org/10.1016/j.bbr.2003.08.014 (2004).

14. Xiong, N. et al. Stereotaxical infusion of rotenone: A reliable rodent model for Parkinson's disease. PLoS ONE 4, e7878 (2009).

15. Normando, E. M. et al. The retina as an early biomarker of neurodegeneration in a rotenone-induced model of Parkinson's disease: Evidence for a neuroprotective effect of rosiglitazone in the eye and brain. Acta Neuropathol. Commun. 4, 86 (2016).

16. Esteve-Rudd, J. et al. Rotenone induces degeneration of photoreceptors and impairs the dopaminergic system in the rat retina. Neurobiol. Dis. https://doi.org/10.1016/j.nbd.2011.06.009 (2011).

17. Zhang, X., Jones, D. \& Gonzalez-Lima, F. Neurodegeneration produced by rotenone in the mouse retina: A potential model to investigate environmental pesticide contributions to neurodegenerative diseases. J. Toxicol. Environ. Heal. Part A Curr. Issues 69, 1681-1697 (2006).

18. Vecino, E., Rodriguez, F. D., Ruzafa, N., Pereiro, X. \& Sharma, S. C. Glia-neuron interactions in the mammalian retina. Prog. Retin. Eye Res. https://doi.org/10.1016/j.preteyeres.2015.06.003 (2016).

19. Newman, E. \& Reichenbach, A. The Muller cell: A functional element of the retina. Trends Neurosci. https://doi.org/10.1016/ 0166-2236(96)10040-0 (1996). 
20. Bringmann, A. et al. Müller cells in the healthy and diseased retina. Prog. Retin. Eye Res. https://doi.org/10.1016/j.preteyeres.2006. $05.003(2006)$.

21. Sorrentino, F. S., Allkabes, M., Salsini, G., Bonifazzi, C. \& Perri, P. The importance of glial cells in the homeostasis of the retinal microenvironment and their pivotal role in the course of diabetic retinopathy. Life Sci. 162, 54-59 (2016).

22. Telegina, D. V., Kozhevnikova, O. S. \& Kolosova, N. G. Changes in retinal glial cells with age and during development of age-related macular degeneration. Biochem. Mosc. 83, 1009-1017 (2018).

23. Rashid, K., Akhtar-Schaefer, I. \& Langmann, T. Microglia in retinal degeneration. Front. Immunol. 10, 1975 (2019).

24. Karlstetter, M. et al. Retinal microglia: Just bystander or target for therapy?. Prog. Retin. Eye Res. https://doi.org/10.1016/j.prete yeres.2014.11.004 (2015).

25. Harada, T. et al. Microglia-Müller glia cell interactions control neurotrophic factor production during light-induced retinal degeneration. J. Neurosci. https://doi.org/10.1523/jneurosci.22-21-09228.2002 (2002).

26. Abcouwer, S. F. Müller cell-microglia cross talk drives neuroinflammation in diabetic retinopathy. Diabetes 66, 261-263 (2017).

27. Wang, M. \& Wong, W. T. Microglia-Müller cell interactions in the retina. Adv. Exp. Med. Biol. 801, 333-338 (2014).

28. Schober, M. S., Chidlow, G., Wood, J. P. M. \& Casson, R. J. Bioenergetic-based neuroprotection and glaucoma. Clin. Exp. Ophthalmol. https://doi.org/10.1111/j.1442-9071.2008.01740.x (2008).

29. Chrysostomou, V., Rezania, F., Trounce, I. A. \& Crowston, J. G. Oxidative stress and mitochondrial dysfunction in glaucoma. Curr. Opin. Pharmacol. https://doi.org/10.1016/j.coph.2012.09.008 (2013).

30. Lee, S. et al. Impaired complex-I-Linked respiration and ATP synthesis in primary open-angle glaucoma patient lymphoblasts. Investig. Ophthalmol. Vis. Sci. https://doi.org/10.1167/iovs.12-9596 (2012).

31. Ai, L. W. et al. Minocycline inhibits LPS-induced retinal microglia activation. Neurochem. Int. https://doi.org/10.1016/j.neuint. 2005.04.018 (2005).

32. Han, X. et al. Tetramethylpyrazine attenuates endotoxin-induced retinal inflammation by inhibiting microglial activation via the

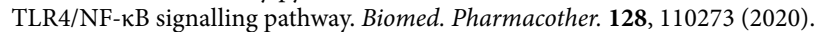

33. Srinivasan, B., Roque, C. H., Hempstead, B. L., Al-Ubaidi, M. R. \& Roque, R. S. Microglia-derived pronerve growth factor promotes photoreceptor cell death via p75 neurotrophin receptor. J. Biol. Chem. https://doi.org/10.1074/jbc.M402872200 (2004).

34. Honjo, M. et al. Expression of ciliary neurotrophic factor activated by retinal Muller cells in eyes with NMDA- and kainic acidinduced neuronal death. Investig. Ophthalmol. Vis. Sci. 41, 552-560 (2000).

35. Eastlake, K., Luis, J. \& Limb, G. A. Potential of Müller glia for retina neuroprotection. Curr. Eye Res. 45, 339-348 (2020).

36. Rutar, M., Natoli, R., Chia, R. X., Valter, K. \& Provis, J. M. Chemokine-mediated inflammation in the degenerating retina is coordinated by Müller cells, activated microglia, and retinal pigment epithelium. J. Neuroinflammation 12, 8 (2015).

37. Garcia, T. B., Hollborn, M. \& Bringmann, A. Expression and signaling of NGF in the healthy and injured retina. Cytokine Growth Factor Rev. 34, 43-57 (2017).

38. Wang, M., Ma, W., Zhao, L., Fariss, R. N. \& Wong, W. T. Adaptive Müller cell responses to microglial activation mediate neuroprotection and coordinate inflammation in the retina. J. Neuroinflammation 8, 1-19 (2011).

39. Lu, Y. Z. et al. Photobiomodulation with $670 \mathrm{~nm}$ light ameliorates Müller cell-mediated activation of microglia and macrophages in retinal degeneration. Exp. Eye Res. https://doi.org/10.1016/j.exer.2017.09.002 (2017).

40. Harada, T. et al. Modification of glial-neuronal cell interactions prevents photoreceptor apoptosis during light-induced retinal degeneration. Neuron https://doi.org/10.1016/S0896-6273(00)81185-X (2000).

41. Sawai, H., Clarke, D. B., Kittlerova, P., Bray, G. M. \& Aguayo, A. J. Brain-derived neurotrophic factor and neurotrophin-4/5 stimulate growth of axonal branches from regenerating retinal ganglion cells. J. Neurosci. 16, 3887-3894 (1996).

42. Cui, Q. \& Harvey, A. R. NT-4/5 reduces naturally occurring retinal ganglion cell death in neonatal rats. NeuroReport 5, 1882-1884 (1994).

43. Harada, C. et al. Role of neurotrophin-4/5 in neural cell death during retinal development and ischemic retinal injury in vivo. Investig. Ophthalmol. Vis. Sci. 46, 669-673 (2005).

44. Liu, R., Wang, Y., Pu, M. \& Gao, J. Effect of alpha lipoic acid on retinal ganglion cell survival in an optic nerve crush model. Mol. Vis. 22, 1122-1136 (2016).

45. Banfield, M. J. et al. Specificity in Trk receptor: Neurotrophin interactions. Structure https://doi.org/10.1016/s0969-2126(01) 00681-5 (2001).

46. Naylor, R. L. et al. A discrete domain of the human TrkB receptor defines the binding sites for BDNF and NT-4. Biochem. Biophys. Res. Commun. https://doi.org/10.1006/bbrc.2002.6468 (2002).

47. Berkemeier, L. R. et al. Neurotrophin-5: A novel neurotrophic factor that activates trk and trkB. Neuron https://doi.org/10.1016/ 0896-6273(91)90287-A (1991).

48. Feng, L. et al. Long-term protection of retinal ganglion cells and visual function by brain-derived neurotrophic factor in mice with ocular hypertension. Investig. Ophthalmol. Vis. Sci. https://doi.org/10.1167/iovs.16-19825 (2016).

49. Domenici, L. et al. Rescue of retinal function by BDNF in a mouse model of glaucoma. PLoS ONE https://doi.org/10.1371/journ al.pone.0115579 (2014).

50. Xu, L., Zhang, Z., Xie, T., Zhang, X. \& Dai, T. Inhibition of BDNF-AS provides neuroprotection for retinal ganglion cells against ischemic injury. PLoS ONE https://doi.org/10.1371/journal.pone.0164941 (2016).

51. Gottmann, K., Mittmann, T. \& Lessmann, V. BDNF signaling in the formation, maturation and plasticity of glutamatergic and GABAergic synapses. Exp. Brain Res. 199, 203-234 (2009).

52. Ip, N. Y. et al. Similarities and differences in the way neurotrophins interact with the Trk receptors in neuronal and nonneuronal cells. Neuron 10, 137-149 (1993).

53. McAllister, A. K., Lo, D. C. \& Katz, L. C. Neurotrophins regulate dendritic growth in developing visual cortex. Neuron 15, 791-803 (1995).

54. Fan, G. et al. Knocking the NT4 gene into the BDNF locus rescues BDNF deficient mice and reveals distinct NT4 and BDNF activities. Nat. Neurosci. 3, 350-357 (2000).

55. Minichiello, L. et al. Point mutation in trkB causes loss of NT4-dependent neurons without major effects on diverse BDNF responses. Neuron 21, 335-345 (1998).

56. Cellerino, A. \& Kohler, K. Brain-derived neurotrophic factor/neurotrophin-4 receptor TrkB is localized on ganglion cells and dopaminergic amacrine cells in the vertebrate retina. J. Comp. Neurol. https://doi.org/10.1002/(SICI)1096-9861(19970915)386:1\% 3c149::AID-CNE13\%3e3.0.CO;2-F (1997).

57. Llamosas, M. M., Cernuda-Cernuda, R., Huerta, J. J., Vega, J. A. \& García-Fernández, J. M. Neurotrophin receptors expression in the developing mouse retina: An immunohistochemical study. Anat. Embryol. (Berl). https://doi.org/10.1007/s004290050053 (1997).

58. Vecino, E., Caminos, E., Ugarte, M., Martín-Zanca, D. \& Osborne, N. N. Immunohistochemical distribution of neurotrophins and their receptors in the rat retina and the effects of ischemia and reperfusion. Gen. Pharmacol. https://doi.org/10.1016/S03063623(97)00361-3 (1998).

59. De Hoz, R. et al. Retinal macroglial responses in health and disease. Biomed Res. Int. 2016, 1-13 (2016).

60. Neta, R., Vogel, S. N., Sipe, J. D., Wong, G. G. \& Nordan, R. P. Comparison of in vivo effects of human recombinant IL 1 and human recombinant IL 6 in mice. Lymphokine Res. 7, 403-412 (1988). 
61. Nagineni, C. N., Detrick, B. \& Hooks, J. J. Synergistic effects of gamma interferon on inflammatory mediators that induce interleukin-6 gene expression and secretion by human retinal pigment epithelial cells. Clin. Diagn. Lab. Immunol. 1, 569-577 (1994).

62. Cheng, S.-C., Huang, W.-C., Pang, J.-H.-S., Wu, Y.-H. \& Cheng, C.-Y. Quercetin inhibits the production of IL-1 $\beta$-induced inflammatory cytokines and chemokines in ARPE-19 cells via the MAPK and NF-кB signaling pathways. Int. J. Mol. Sci. Artic. J. Mol. Sci. 20, 2957 (2019).

63. Elner, V. M. et al. Interleukin-6 (IL-6) gene expression and secretion by cytokine-stimulated human retinal pigment epithelial cells. Exp. Eye Res. 54, 361-368 (1992).

64. Fischer, A. J., Zelinka, C. \& Milani-Nejad, N. Reactive retinal microglia, neuronal survival, and the formation of retinal folds and detachments. Glia 63, 313-327 (2015).

65. Todd, L. et al. Reactive microglia and IL1//IL-1R1-signaling mediate neuroprotection in excitotoxin-damaged mouse retina. J. Neuroinflammation 16, 118 (2019).

66. Hicks, D. \& Courtois, Y. The growth and behaviour of rat retinal Müller cells in vitro 1. An improved method for isolation and culture. Exp. Eye Res. https://doi.org/10.1016/0014-4835(90)90063-Z (1990).

67. Pereiro, X., Ruzafa, N., Acera, A., Urcola, A. \& Vecino, E. Optimization of a method to isolate and culture adult porcine, rats and mice Müller glia in order to study retinal diseases. Front. Cell. Neurosci. https://doi.org/10.3389/fncel.2020.00007 (2020).

68. Maekawa, S. et al. The neuroprotective effect of hesperidin in NMDA-induced retinal injury acts by suppressing oxidative stress and excessive calpain activation. Sci. Rep. 7, 6885 (2017).

69. Tawarayama, H., Feng, Q., Murayama, N., Suzuki, N. \& Nakazawa, T. Cyclin-dependent kinase inhibitor 2B mediates excitotoxicityinduced death of retinal ganglion cells. Investig. Ophthalmol. Vis. Sci. 60, 4479-4488 (2019).

70. Rodriguez, A. R. \& Luis, P. The RNA binding protein RBPMS is a selective marker of ganglion cells in the mammalian retina. J. Comp. Neurol. 1443, 1411-1443 (2014).

\section{Acknowledgements}

We thank Drs. Kazuhiko Namekata (Tohoku University, Japan) and Takayuki Harada (Tokyo Metropolitan Institute of Medical Science, Japan) for providing for instructing on mouse Müller cell preparation. We thank Junko Sato and Rieko Kamii for technical assistance with the experiments. This work was supported by JSPS KAKENHI Grant Number JP18K09435.

\section{Author contributions}

H.T. and T.N. designed the study. H.T., M.IY. and N.H. performed the experiments. All the authors analyzed the data. H.T. and M.IY. wrote the paper.

\section{Competing interests}

The authors declare no competing interests.

\section{Additional information}

Supplementary Information The online version contains supplementary material available at https:/doi.org/ 10.1038/s41598-021-90604-w.

Correspondence and requests for materials should be addressed to T.N.

Reprints and permissions information is available at www.nature.com/reprints.

Publisher's note Springer Nature remains neutral with regard to jurisdictional claims in published maps and institutional affiliations.

(c) (i) Open Access This article is licensed under a Creative Commons Attribution 4.0 International License, which permits use, sharing, adaptation, distribution and reproduction in any medium or format, as long as you give appropriate credit to the original author(s) and the source, provide a link to the Creative Commons licence, and indicate if changes were made. The images or other third party material in this article are included in the article's Creative Commons licence, unless indicated otherwise in a credit line to the material. If material is not included in the article's Creative Commons licence and your intended use is not permitted by statutory regulation or exceeds the permitted use, you will need to obtain permission directly from the copyright holder. To view a copy of this licence, visit http://creativecommons.org/licenses/by/4.0/.

(C) The Author(s) 2021 